\title{
Structuralism and Its Ontology
}

\author{
MARC GASSER \\ Harvard University
}

A prominent version of mathematical structuralism holds that mathematical objects are at bottom nothing but "positions in structures," purely relational entities without any sort of nature independent of the structure to which they belong. Such an ontology is often presented as a response to Benacerraf's "multiple reductions" problem, or motivated on hermeneutic grounds, as a faithful representation of the discourse and practice of mathematics. In this paper I argue that there are serious difficulties with this kind of view: its proponents rely on a distinction between "essential" and "nonessential" features of mathematical objects, and there's no good way to articulate this distinction which is compatible with basic structuralist commitments. But all is not lost. For I further argue that the insights motivating structuralism (or at least those worth preserving) can be preserved without formulating the view in ontologically committal terms.

$\mathrm{M}$ ATHEMATICAL structuralists think that mathematics is fundamentally concerned with structures, or with the relations mathematical objects bear to each other in virtue of belonging to some structure. This thought is often developed as an ontological claim: mathematical objects are said to be mere "positions in structures," fundamentally relational entities without any internal composition or any sort of nature independent of the structure to which they belong. ${ }^{\mathrm{I}}$

Structuralists typically motivate such an ontology on philosophical and hermeneutic grounds. On the philosophical side, the structuralist ontology is often presented as a response to the "multiple reductions" problem raised in Benacerraf ( 1965 ). ${ }^{2}$ On the hermeneutic side, the structuralist ontology is said to be faithful to the discourse and practice of mathematics-mathematical discourse seems to

I. Major proponents of structuralism as an ontological view include Resnik (I98 I; I997), Parsons (I990; I995), and Shapiro (I997). I'll briefly discuss other forms of structuralism in what follows.

2. See for instance Parsons (I990: 306-II), Resnik (I997: 91, 267), and Shapiro (I997: 5-6, $78-80)$. 
refer to mathematical objects, and mathematicians only seem to care about the structural relations these objects bear to one another. ${ }^{3}$

I'm going to argue for two claims in this paper. The first is that there are serious difficulties with the structuralist ontology. In particular, I'll argue that any precise articulation of such an ontology rests on a distinction between "essential" and "nonessential" features of mathematical objects, and that there's no satisfying way to spell out this distinction which is compatible with basic structuralist commitments. The second claim is that the insights motivating structuralism are either not worth preserving, or are such that they can be preserved without formulating the view in ontologically committal terms. My argument here will have two parts. First I'll try to show that Benacerraf's problem is, upon closer inspection, poor motivation for a structuralist ontology (and indeed for any kind of mathematical ontology). Then I'll argue that the hermeneutic insights mentioned above can be preserved on an ontologically neutral version of structuralism that avoids the difficulties faced by its ontologically committal counterparts.

If this is correct, structuralism will emerge a more modest view than many of its current proponents suggest.

\section{Structuralist Insights}

Mathematical structuralism, as I'll be discussing it in this paper, is a view concerning the subject matter of mathematics. At first approximation, the view states that mathematics is the investigation of certain structures, and that mathematical objects can be characterized by the structural relations they bear to each other. A contrast is often drawn with commonplace physical entities: we might expect an analysis of some physical entity to reveal its composition, or molecular structure, or mass, but (on the structuralist view) it would be a mistake to expect a deeper analysis of numbers or sets to reveal anything important beyond the relations they bear to other numbers or sets.

This basic idea has been developed in two ways in the recent literature. 4 The first approach, eliminative structuralism, is motivated by the thought that ob-

3. See for instance Parsons (2004: $\int 2$ ) and Shapiro (2008: 289, 300-30I).

4. The following distinction between eliminative and noneliminative structuralism follows Parsons (see for instance I990; or 2004: 57-59). It corresponds roughly to Shapiro's distinction between in re and ante rem structuralism (I997: I49-50), Hale's distinction between pure-structuralism and abstract-structuralism (I996: I25), and Dummett's distinction between hard-headed and mystical structuralism (I99I: 295-96). There are subtle differences in each case, but they won't matter for the purposes of this paper. 
jects with a purely structural nature are deeply problematic, and that they really shouldn't count as objects at all. Eliminative structuralists seek to paraphrase statements referring to mathematical objects into general statements about what holds in any collection of objects satisfying certain conditions. ${ }^{5}$ Such paraphrase saves the eliminative structuralist from referring to mathematical objects-and indeed from referring to any particular structure to which these objects might belongand thereby frees her from ontological commitments she deems problematic.

Noneliminative structuralism, by contrast, doesn't shy away from an essentially relational conception of mathematical objects. Ordinary mathematical statements are taken at face-value, as statements with singular terms denoting objects like triangles, numbers, sets, and so on-it's just that the nature of these objects is constituted by the structural relations they bear to one another, and nothing more. If these objects seem metaphysically queer, so be it.

My argument in what follows will only concern structuralism of the noneliminative variety-the kind of structuralism which officially endorses a certain conception of mathematical objects. I'll also focus my attention on comprehensive structuralist views, that is, structuralist views applying to all mathematical objects. This is partly because most recent proponents of structuralism endorse it in its comprehensive form, ${ }^{6}$ and partly because more limited forms of structuralism are much less interesting. Indeed, it seems uncontroversial that in certain subfields mathematicians define objects purely for the sake of their structural features (group theory being a paradigmatic example). But such definitions typically involve an appeal to set theory as a background theory. As I see it, structuralism gets its bite precisely by applying the view taken in such "algebraic" subfields to disciplines like set theory itself, where objects aren't usually introduced by construction from some further background theory.

From now on, then, I'll use "structuralism" to denote noneliminative, comprehensive structuralism.

5. So a statement like " $5+2=7$ " might be read as elliptical for "in any $N, \mathrm{O}, \mathrm{I},+, \times$ satisfying Peano's axioms, $5+2=7$," or, on a more sophisticated eliminativist reading, for the conjunction stating that it is logically possible that there be a structure satisfying Peano's axioms, and that " $5+2=$ 7 " holds in any possible structure of this type (where the interpretation of the terms in the equation depends on the structure). I believe the latest efforts in this direction are in Hellman (2005).

6. This hasn't always been the case. In Dedekind (I 888), for instance, statements of arithmetic are characterized as implicitly general statements about all simply infinite systems satisfying certain structural conditions, but these systems are not further explained in structuralist terms. The Bourbaki group can also be seen as trying to develop a structuralist account of mathematics from within (nonstructuralist) set theory, and it has been suggested that category theory might play a similar role (cf. Bell I98 I; I986). For a more recent defense of a restricted form of structuralism, see Linnebo (2008). 


\section{Why Be a Structuralist?}

Structuralism is often motivated on philosophical grounds, as a response to the "multiple reductions" problem raised in Benacerraf (I965).7 The problem arises in the context of set theoretic developments (or "reductions") of arithmetic: as is well-known, we can interpret arithmetic in set theoretic terms by identifying the natural number sequence with certain sets. But in fact many such interpretations are possible, and there seems to be no good reason to choose one over the other. One might, for instance, use von Neumann ordinals and identify the number 2 with $\{\varnothing,\{\varnothing\}\}$, or use Zermelo numerals and identify the number 2 with $\{\{\varnothing\}\}$. Benacerraf argues that since numbers can't be identified with particular sets, they aren't sets at all. He further argues, along similar lines, that numbers aren't objects: given any system of objects which might be identified with the natural number sequence, other systems exist that do the job equally well-for as Benacerraf observes, all that matters for the purposes of reduction is the preservation of the structural relations between members of the natural number sequence.

The structuralist response is to accept Benacerraf's observation but deny his conclusion: it's true that only the structural relations between natural numbers matter, and yet it's a mistake to conclude that natural numbers aren't objects. ${ }^{8}$ On the structuralist view, natural numbers are positions in an abstract structure (the structure of an $\omega$-sequence) which various systems of objects might instantiate. Such positions are objects, even if their identity only really depends on the relations they bear to each other. The structuralist ontology is thus meant to provide a response to Benacerraf's problem-or perhaps a dissolution of the problem, since on the structuralist view there's really no problem to begin with.

Structuralism has also been motivated on hermeneutic grounds. Indeed, mathematical discourse is (on the face of it) about mathematical objects, and working mathematicians typically seem indifferent to questions concerning these objects' nonstructural features: they rest content having described natural numbers as members of an $\omega$-sequence; real numbers as members of a complete ordered field; or planes, points, and lines as members of a structure satisfying the basic axioms of geometry. In other words, the issue never arises whether natural numbers are really to be identified with von Neumann ordinals or Zermelo numerals, or whether real numbers are really to be identified with equivalence classes of Cauchy sequences or Dedekind cuts-what matters is only that some structure with the right properties

7. Benacerraf's article has become something of a locus classicus for the problem, but as Parsons notes the existence of such reductions was already well-known to structuralists at the turn of the twentieth century (I990: 304). Though Benacerraf focuses on numbers, the difficulty is easily extended to any mathematical object

8. This is not the response of eliminative structuralists, who do endorse Benacerraf's conclusion (not always for the same reasons). 
has been shown to exist. ${ }^{9}$ The structuralist has a good explanation for this indifference: questions concerning the nonstructural identity of mathematical objects are irrelevant because there is, at bottom, no nonstructural identity to be found.

\section{Defining Structuralism}

I've so far presented the structuralist view in rather informal terms, invoking various slogans concerning "essentially relational" objects, whose natures or identities are given by the structural relations they bear to each other. But what exactly are these structural relations? In what sense do they "determine the nature" of mathematical objects, and is it really necessary to invoke any robust distinction between "essential" and "nonessential" features to articulate this kind of view?

The first of these questions is usually answered as follows. Suppose we have some system of objects related to each other in certain ways. A structure is what remains once we abstract away any features these objects might have outside their relations to each other. So for instance, we might abstract the structure of an $\omega$ sequence from the system of finite von Neumann ordinals $\left\langle\omega, \emptyset, S_{\mathrm{V}}(x)=x \cup\{x\}\right\rangle$, or from the system of Zermelo numerals $\left\langle\omega, \emptyset, S_{Z}(x)=\{x\}\right\rangle$, or from the system of even positive integers $\left\langle 2 \mathrm{~N}, \circ, S_{\mathrm{E}}(n)=n+2\right\rangle$, or indeed from any system of objects with a distinguished first element closed under an inductive successor relation. ${ }^{\text {IO }}$ In this case, the resulting structure will just be a collection of positions related to each other by an abstract successor relation. ${ }^{\text {II }}$ Relations of this sort-that is, relations we find instantiated in various systems of some isomorphism-type-count as structural relations, and any feature definable from these relations counts as a structural property. So for instance "being odd" and "being positive" count as structural properties of the natural numbers, while properties like "being the number of fingers on my hand" or "being mind-independent" do not.

Now, one might well wonder what sorts of objects these abstracted structures are supposed to be. And as far as I can tell there's no consensus on this point. Resnik only invokes structures as an analogy, stating that in arithmetic we treat numbers "as if they were positions in patterns" (1997: 250). Shapiro develops a

9. A common way of putting this point is to say that mathematicians only care about things "up to isomorphism."

Io. We need not specify here exactly how this abstraction procedure is meant to happen, for structuralists typically don't claim that their theory holds any epistemological advantage over more classical forms of Platonism.

II. Structuralists say structures are exemplified by or realized by or instantiated in various systems of objects. For instance, the successor relation belonging to the structure of an $\omega$-sequence is exemplified by $S_{\mathrm{V}}(x)$ in the finite von Neumann ordinals, and by $S_{\mathrm{Z}}(x)$ in the Zermelo numerals. The number $\circ$ is exemplified by $\varnothing$ in both of these systems, and by $\circ$ in the system of even positive integers. 
full-blown, axiomatized ontology of structures, designed on the model of ante rem universals, while Parsons seeks to avoid such an axiomatic development by semantic ascent, that is, by introducing certain predicates and functors to talk of "structures" at a metalinguistic level, rather than introducing structures as new objects in our ontology. ${ }^{\mathrm{I} 2}$ For my purposes it won't matter which option is chosen, as long as structures are taken to exhibit the relations instantiated in any system of objects of some type, and these relations are taken to determine the nature of the structure's objects. All the above structuralists should agree on this point.

But in what sense do these structural properties "determine the nature" of mathematical objects? One flatfooted response would be that they exhaust the properties one can meaningfully ascribe to mathematical objects. ${ }^{\mathrm{I} 3}$ But as many structuralists have noted, this is clearly false-whatever one might wish to say about the applications of mathematics, assertions like " 2 is the number of extant alligator species" or "67 is the number of moons of Jupiter" surely aren't nonsense, even though the relevant properties aren't structural. So a more subtle distinction between essential and nonessential properties of mathematical objects is necessary to spell out the structuralist view: it won't do to claim mathematical objects only have structural properties, or that these are the only properties they could coherently be said to possess.

In what other sense could an object's structural features be essential, or naturedetermining? Structuralists have very little to say on this point. It's sometimes claimed that mathematical objects are fundamentally incomplete-that they "have no more of a 'nature' than is given by the basic relations of a structure to which they belong" (Parsons 2004: 57), or that "the essence of a natural number," for instance, "is its relations to other natural numbers" (Shapiro I997: 72, emphasis in the original), or that "every property that 2 enjoys comes in virtue of its being [the second] place in the natural number structure [...] because that is what 2 is" (Shapiro 2006: I2I). But this isn't much help, because it's never made clear how we're meant to understand the talk of "natures" and "essences" in such slogans.

I 2. So for instance where Shapiro seeks to introduce an $\omega$-sequence structure meeting certain formal requirements, Parsons will merely describe the ways we use a predicate "N" and some functors like " +" or " $x$ " (assuming here that " $\circ$ " and " $S$ " are defined in terms of these functors). The idea behind Parsons' approach is that we can describe the way such functors and predicates operate at an informal metalinguistic level, without introducing any of the entities to which they might refer. (The aim here is to avoid an axiomatic system in which structures are essentially treated like sets, and so seem like they should themselves be subject to further structuralist treatment, making the view rather circular.) Shapiro's ontology is developed in (1997: 90-97). Parsons raises some difficulties for Shapiro's approach and defends semantic ascent in (2004: 64-66) and (2008: I I I-I4).

I3. This line is suggested by some remarks of Parsons ("there is only a certain specific range of predicates such that there is a fact of the matter as to whether they are true of the object in question") (I990: 334) and Shapiro ("The number 2, for example, is no more and no less than the second position in the natural-number structure; 6 is the sixth position") (I997: 72). But in fact both authors reject such a flatfooted response (cf. Parsons 2004: 57; Shapiro 2008: 286). 
Most prominent structuralists have been reluctant to advance any positive view concerning essences, and their reluctance makes structuralism a somewhat elusive target. Critics will deny that mathematical objects are "essentially" or "fundamentally" relational by assuming some interpretation of essentialist talk and presenting examples of nonstructural properties that count as "essential" on this interpretation (see for instance Hellman 200I: I92-94; or Linnebo 2008), and structuralists then respond by denying some of their critics' assumptions about essences (cf. Parsons 2004: 72-74; and Shapiro 2008: 303-04).

I won't be dwelling too much on these past criticisms. As I see it, they all fail to touch the strongest formulation of the structuralist view, which I'll be presenting below. But it will be worthwhile to review them before moving on, if only to remind ourselves why common interpretations of essentialist talk don't sit well with structuralism's basic commitments.

\section{I. The Counterfactual Path}

One might think structuralist claims about essences can be explained in counterfactual terms, for instance by saying that an object's essential properties are those it has in all possible worlds. ${ }^{\mathrm{I}}$ The structuralist view would then be that all and only the structural properties of mathematical objects are constant across all possible worlds. For instance, 2 is the successor of I (and I + I, and positive, and so on) in every world, but there are worlds where it fails to be the number of extant alligator species. So far so good.

But this approach is too permissive for the structuralist's purposes. For as many critics of structuralism were quick to note, there's no world in which mathematical objects fail to be abstract, though "being abstract" is not a structural property. ${ }^{\text {I5 }}$ Even with basic counting applications of the natural numbers, the case may not be as clear as it seems: 2 might indeed fail to count extant alligator species in some world, but it will always bear certain nonstructural relations to other mathematical objects-for instance 2 has, at every world, the property of being the number of objects in $\{\omega, \omega+\mathrm{I}\}$. But this property, necessary though it is, isn't definable using the successor relation alone, and therefore shouldn't count as essential on a structuralist account of the natural numbers.

Now structuralists may well insist that properties like abstractness, while not structural stricto sensu, nonetheless follow from the structural character of math-

I4. Or in all possible worlds in which it exists-I take it these amount to the same thing in the case of mathematical objects.

I 5. See for instance Linnebo (2008: 65). As Shapiro notes, even if one denies that mathematical objects are necessarily abstract, it seems right to say that they need not have a concrete instantiation, and if this is indeed right the (nonstructural) property "being possibly abstract" would serve as a counterexample (2006: I20). 
ematical objects in some straightforward way (and likewise for properties stemming from the counting applications of numbers). That is, maybe abstractness is a feature mathematical objects possess in virtue of their structural natures, and one which should qualify as "essential" on that account, even if it isn't definable in structural terms. ${ }^{\mathrm{I} 6}$ But this is just to say that structural properties are "basic" or "fundamental" in some other, non-counterfactual sense, and leads us back to our initial problem: how exactly are structural properties fundamental, and properties like abstractness derivative? If we want to claim that an object's abstractness "follows" from its structural properties, we should have some story to tell about the sense in which certain attributes of mathematical objects might "follow from" or hold "in virtue of" other, more basic ones.

\subsection{The Fundamentalist Path}

Or should we? Maybe the right move is to not seek an account for our talk of essences or priority, and simply take these notions as primitives. The structuralist view could then be stated in a number of ways-one might say numbers, sets, and so on are all the objects they are in virtue of their structural properties, or one might say that structural properties ground, or are fundamental to, or explain (in a metaphysical sense) their identity, where these formulations would all be taken to express a primitive notion of essence. ${ }^{\mathrm{I} 7}$

But this response leaves structuralism open to another well-known line of criticism. For if the fundamental identity of individual mathematical objects is given by their structural relations, it's very natural to think that these structural relations would also be sufficient to distinguish mathematical objects from each other. It's natural to think this because a complete account of some individual's fundamental identity is supposed to tell us what it is to be that individual, that is, to answer a Socratic "What is $X$ ?" question. ${ }^{\text {I8 }}$ And if the answer to the Socratic questions "What is $X$ ?" and "What is $Y$ ?" is the same, then $X$ and $Y$ can plausibly be taken to be the same thing. To take a simple example, if the fundamental identity of physical objects is given by certain arrangements of particles, then it seems plausible that objects constituted by the exact same particles arranged in exactly the same

I6. Shapiro says something along these lines (2006: I2I).

I7. It's beyond the scope of this paper to examine notions of grounding or essence in any sort of detail. I have in mind here a notion similar to the one defended in Fine (I994; 200I). In what follows I'll use its various formulations interchangeably-for my purposes, all that matters is that we agree that an object's essence (or the features which ground, or are fundamental to, or explain its identity) is what makes it the object it is. I take it this is part of the standard understanding of metaphysical essence.

I 8. For the connection between grounding notions and Socratic questions of this sort, see Rosen (2010: I22). 
manner are the same. So in the mathematical case we'd also expect that all objects sharing the same essential features be identical. ${ }^{19}$

But as Burgess and Keränen pointed out, structural relations are insufficient to distinguish conjugate elements of nonrigid structures. ${ }^{20}$ The complex numbers $i$ and $-i$, for instance, share all their relations in $\mathrm{C}$, as do $\mathrm{I}$ and $-\mathrm{I}$ in the additive group $(Z,+)$ and any two points in the Euclidean plane. Thus they will share all their relations in the abstracted structures of which these are instances. But then it's not clear how structuralists distinguish $i$ from $-i$ if these objects share all their structural features, and these features are the only ones grounding their identity.

This isn't a knock-down objection, since structuralists can deny the natural thought I voiced above, which links the essence of mathematical objects to the identity conditions for these objects. For instance, one might argue that the distinctness of mathematical objects isn't really based on anything, and that it, too, should be taken as an unanalyzed primitive rather than subject to metaphysical treatment. Or one might simply give up on some of structuralism's explanatory burdens: if one doesn't expect an ontology of mathematics to explain facts about individuation, then the Burgess-Keränen objection will, of course, sound hollow. ${ }^{2 \mathrm{I}}$

I9. I'm only claiming here that we'd expect this without any further discussion of essences. In what follows I'll be considering the response that this natural thought must simply be false in the mathematical case (and perhaps also in the case of physical objects).

20. Structures are nonrigid if they have nontrivial automorphisms. By conjugate elements I mean the elements exchanged by some nontrivial automorphism. The objection was initially voiced in Burgess (I999: 287-88), and developed in detail in Keränen (200I). See also Keränen (2006).

2I. You might think a less radical response is possible, for rejecting the natural thought only requires that distinct mathematical objects have some non-essential property that would allow us to distinguish them. For instance, there is an irreflexive relation holding between $i$ and $-i$ in $\mathrm{C}$, namely the relation " $x$ is a nonzero additive inverse of $y$," and if we think the existence of such an irreflexive relation is sufficient to establish nonidentity, we will be in a position to distinguish all the problematic pairs of conjugates mentioned above (this is the suggestion advanced in Ladyman 2005). But in fact this more subtle response doesn't generalize: in the following three directed graphs, for instance, the $a$ and $b$ nodes share all their properties:

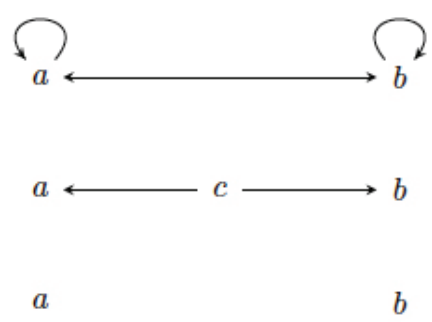

In the first case, $a$ and $b$ are pointed to by everything, in the second they're both pointed to only by $c$, and in the third they're pointed to by nothing at all. In all these cases they should count as distinct nodes of the graph, yet in none do we have any irreflexive relation (outside nonidentity, of course) which would serve to distinguish the two (similar graphs are invoked in Keränen 200I: 32I; Button 
I won't dwell on the particular merits of these responses-my point here is only that the structuralist has to reject the natural thought I presented above, and that this runs against our basic intuitions concerning a primitive notion of essence. So it won't do to appeal to some intuitive notion of essence, or to invoke the notion of essence which is often used in metaphysical accounts of other, nonmathematical domains, and leave things at that. Structuralists have to say more about their interpretation of essentialist talk.

I see two options. The structuralist can simply dig her heels: if some mathematical cases don't sit well with our broader conception of essence, then maybe that conception needs to be revised, or maybe a different notion of essence is at play in the mathematical cases. This was, after all, supposed to be a primitive notion of essence, so why not stipulate that mathematical essences are quite different from those that show up in other domains? Yet without any positive account of mathematical essences, this sort of stipulation seems purely ad hoc. It's not clear how one could argue against it, except to point out that the structuralist's primitive notion of essence should earn its keep, and that so far it hasn't been motivated except as a response to the sort of objection voiced in this section. ${ }^{22}$

The better answer, I think, is to claim that the structuralist's notion of essence explains certain key features of mathematical practice, and that its use is warranted by its explanatory fruitfulness. But on such a view our interpretation of essentialist talk would be determined by the practice and discourse of working mathematicians. And if that's the case it's hard to see what would be gained by taking the notion of essence as a metaphysical primitive rather than formulating structuralism as more of an empirically-grounded hypothesis.

In fact, thinking of structuralism this way quickly leads to what I take to be

2006: 2I 8; Ketland 2006: 309; and Leitgeb and Ladyman 2008: 39I-92). The moral is that we can't dismiss the Burgess-Keränen objection by claiming that it rests on an overly strong version of the Identity of Indiscernibles. So the more radical response I've presented above (identity facts are not grounded in anything, or at least not explained by the ontology of mathematics) is necessary. (This more radical response is presented in Ketland 2006, and Leitgeb and Ladyman 2008, though the idea behind it was already in Parsons 2004: 75, where it's attributed to Linnebo. Shapiro endorses it in 2008: 287-89.)

22. One might object that there has been an attempt to motivate a very thin, non-individuating notion of mathematical essence-for it has been pointed out that a similarly thin notion of essence is sometimes thought to apply to physical objects (cf. for instance Ladyman 2005; Leitgeb and Ladyman 2008: 395-96). But in fact it's a contentious question whether or not physical entities should count as objects when they can't be individuated on the basis of some irreflexive relation (and recall that in the mathematical case, distinct objects need not satisfy any irreflexive relation, as noted in fn.2I). So it's not as though the structuralist's notion of essence can be motivated on the basis of some well-accepted claims about physical objects. In fact, the argument advanced by Leitgeb and Ladyman goes the other way around: claims about the individuation of physical entities are motivated by claims about the distinctness of structural mathematical objects, and these latter claims are supposed to be motivated by mathematical practice (2008: 39I-92). This is precisely the "better answer" I'll be investigating section 3.3 . 
the strongest formulation of the view, which I'll be presenting more fully in this next section.

\subsection{The Path Less Metaphysical}

I've argued so far that structuralists are committed to some distinction between essential and nonessential properties of mathematical objects, and that two common ways of interpreting this distinction leave them open to familiar lines of criticism. A common response to the criticism I discussed rests on a primitive notion of mathematical essence, but the strongest motivation for introducing such a primitive stems from its ability to explain key features of mathematical practice. If this is right, then it seems preferable to draw the distinction between essential and nonessential properties on the basis of mathematical practice alone, rather than arguing about competing intuitions concerning metaphysical essences.

To my knowledge this deflationary option hasn't been explicitly advocated by any prominent structuralist, ${ }^{23}$ but it seems to me the best way to defend the structuralist view. Recall that one source of motivation for structuralism is that it captures a certain indifference exhibited by working mathematicians-so conformity with mathematical practice is taken as a desideratum from the start. Moreover, structuralists often portray themselves as defending a sober, clear-headed understanding of mathematical discourse against dubious metaphysical criticism. I take it this would go hand-in-hand with a similar understanding of mathematical essences, as stemming from nothing more than the role certain features of mathematical objects play in the practice of working mathematicians.

One might argue, then, that structural properties are essential merely in the sense that they're the ones mathematicians care about in their work. So nonstructural properties like "being abstract" or "being the atomic number of boron" would be inessential merely because they don't reflect any mathematically significant fact. Aside from clarifying the structuralist view, such a deflationary approach might serve to manage expectations concerning discernibility. For if mathematical objects have structural "essences" only in the sense that their structural properties are those mathematicians care about, it isn't clear we should expect any identity conditions to follow from their essential properties. One might think, for instance, that identity conditions are simply presupposed by mathematical practice, and that it would therefore be a mistake to expect them to follow from the properties mathematicians focus on in their work. ${ }^{24}$

One final advantage: the deflationary approach makes structuralism something of an empirical thesis. If mathematicians really only care about the structural

23. Though see below for some suggestive remarks Parsons makes in this direction (cf. also Resnik I997: 268-70).

24. As does Shapiro (2008: 293).

Ergo $\cdot$ vol. 2, no. I $\cdot 2015$ 
properties of the objects they study, then such is the nature of these objects-no metaphysics or Leibnizian insight necessary. And if things turn out otherwise, so much the worse for structuralism.

In what follows I'm going to argue that things do in fact turn out otherwise. But a bit of clarification is needed beforehand. The hypothesis under consideration is that structural properties are essential because they're mathematically significant. Clearly, how plausible this is will depend on what we mean by "mathematically significant." Consider for instance basic arithmetic. It's true, of course, that arithmetic can be studied with complete disregard for any of its applications in counting objects, and that in this sense nonstructural properties like "being the number of moons of Jupiter" are dispensable from arithmetic. But that doesn't mean that such applications are completely insignificant. For one thing, they might be thought to motivate the axioms of arithmetic, at least if one thinks that these axioms are partly assessed based on their deductive consequences, and that part of the consequences we wish them to have stem from the use of numbers in counting. In any case, it's hard to imagine not using natural numbers to count things-even once we've agreed on our axioms and engage in pure mathematics, we'll want to talk about an equation having three solutions, or a function taking on some value five times, or a number having seven factors. In all such cases we rely on numbers regarded as cardinal numbers, not just as positions in an $\omega$-sequence. ${ }^{25}$

Now, structuralists might reply that these applications are all well and good, and that cardinal numbers may indeed play some mathematical role, yet insist that they remain insignificant from a foundational perspective-that is, that their cardinal use doesn't reflect any feature one would have to preserve when developing arithmetic. Parsons seems to endorse a position similar to this one in the following passage:

[The] structuralist understanding of arithmetic belongs to sophisticated mathematics; it is not part of the layman's understanding of arithmetic or even the mathematician's before a certain amount of foundational reflection has been undertaken. (2008: 77)

So a more careful version of our hypothesis might be that structural properties are those which play a significant role in sophisticated, foundationally-informed mathematics. This would rule out not only properties like "being the number of moons of Jupiter" or "being the atomic number of boron," which play no mathematical role whatsoever, but also any nonstructural feature playing a dispensable role from

25. This is a point Dummett forcefully presses against structuralists (I99I: 52-54). 
foundationally-informed mathematics. Numbers might help us count factors and equations, for instance, but no mathematician's research would really be doomed if their use as cardinals had to be given up. By contrast, any mathematician invoking "natural numbers" in her research which fail to instantiate the structure of an $\omega$-sequence would fare rather poorly-for she would be mistaken about a key feature (the key feature, structuralists insist) that any proper foundational treatment of arithmetic must aim to secure. And it's in this sense that structural properties are mathematically significant, while counting applications are not. ${ }^{26}$

Yet even if we restrict ourselves to the practice of mathematics informed by foundational considerations - to sophisticated mathematics-it doesn't seem to be the case that mathematicians always disregard nonstructural properties. I'll now turn to two cases where nonstructural properties of mathematical objects seem to have played a significant foundational role. ${ }^{27}$

\subsection{Weyl}

During the course of the nineteenth century it was discovered that problems about elliptic functions could be approached by defining these functions on Riemann surfaces, rather than the standard complex plane. ${ }^{28}$ The elliptic functions in question are the same on either definition, and picking one or the other wouldn't make any difference to what we can prove about them. Thus from a structuralist point of view, it's an idle matter which definition you pick-the choice just amounts to different ways of looking at the same structural relations, as embedded in different systems.

Still, Riemann surfaces are widely considered to be the "right setting" for the investigation of such functions. A natural thought here is that these surfaces are the "right setting" because of their heuristic value, or simply because they provide a more convenient setting to study certain functions (which is what Shapiro I997:

26. Understanding mathematical significance this way might serve to defend the deflationary structuralist against the sort of criticism raised by Weaver (I998), who points out that mathematicians are often interested in non-structural properties of certain "concrete" representations of isomorphic abstract systems. For one might agree that these properties are interesting and useful heuristics, but deny that mathematicians ever thought they played an interesting foundational role.

27. My aim in bringing up these cases isn't just to argue by counterexample: I think they illustrate a relatively widespread concern for nonstructural features of mathematical objects, though this concern is often obscured by the difficulties involved in describing the nonstructural features which matter to mathematics, and explaining how they might matter. I'll be arguing below (in section 4.2) that this kind of concern is compatible with the indifference working mathematicians often display towards the nonstructural features of the objects they study. For now my aim is only to cast some doubt on the idea that the distinction between essential, structural features and their inessential, nonstructural counterparts is simply something afforded by mathematical practice.

28. Riemann surfaces can be seen as a generalization of the complex plane. For more on this historical development, see Wilson (I992: I 50-5I). 
8I suggests), or that they only matter in the context of unsophisticated, foundationally uninformed mathematics. But this is clearly not how Weyl saw the matter:

Every now and then one still hears the opinion that the Riemann surface is nothing more than a "metaphor," nothing more than a tool employed to visualize and illustrate the ambiguity of functions (a very useful and suggestive tool, it is usually conceded). ${ }^{29}$ But this is getting things completely wrong. The Riemann surface is an indispensable, objective component of the theory [of analytic functions]-it serves, in fact, as the foundation for the theory. Moreover, it is not something we might somehow extract from the analytic functions a posteriori; it must be regarded from the start as the native soil in which the functions can grow and thrive. (I9I3: vi-vii, emphasis in the original, translation mine)

Weyl's remarks seem to me to provide some evidence against our empirical hypothesis. Elliptical functions have exactly the same structural properties when embedded in Riemann surfaces as they do when embedded in the complex plane, but presenting them on a Riemann surface isn't just some insignificant matter-if we're to believe Weyl, the embedding is of central foundational importance.

Now, one might object that Weyl's remarks shouldn't be taken too seriously. Perhaps Weyl was simply mistaken about the role played by Riemann surfaces, or perhaps he was overstating their significance. But I don't think this response is open to our deflationary structuralist_-one can't claim to be defending a sober reading of mathematical practice and ignore cases where the practice doesn't conform with one's hypothesis. At the very least the deflationary structuralist owes us an account about the parts of mathematical practice she takes to be significant, and why it might be safe to ignore Weyl's foundationally-informed treatment of Riemann surfaces, where nonstructural properties do seem to play an indispensable role.

One possible argument in this direction would be that Weyl was concerned with the "conceptual" foundations of complex analysis, in the tradition of Riemann and Poincaré, rather than its logical foundations-that is, that Weyl was concerned with providing a setting in which the behavior of certain functions might be understood and fruitfully studied, rather than simply trying to establish their existence and secure the truth of theorems about them. This may be the case, but one would still want to know why logical foundations are the ones relevant to the essence of mathematical objects. ${ }^{3 \circ}$ Anyway I now turn to a case where nonstructural properties do seem to have played a significant justificatory role.

29. The ambiguity in question stems from the formulas describing ellipticals, which suggest ambiguous, one-to-many "functions."

30. The view that "fruitfulness" and "understanding" are purely subjective or psychological notions which only matter in the "context of discovery" doesn't seem generally compelling—at least 


\section{3·3.2. Gödel}

Set theory is usually introduced by talking about the iterative conception of sets. On the iterative conception, sets are formed in progressive stages, as totalities "built up" from elements introduced at an earlier stage. This account is usually presented informally and used to motivate the axioms of ZFC, at least in the sense that it offers an intuitive picture of the universe of sets $V$ that these axioms are meant to describe, and maybe gives us some reason to think this universe is coherent.

Now note that there is more to the sets suggested by the iterative conception than to the sets construed by a structuralist. For instance, the notion that sets are "built up" from certain elements suggests a form of dependence absent from a purely structuralist point of view-on the iterative conception, a set's elements must somehow "already be there" for the set to be formed, while the structuralist view says nothing at all about the dependence of sets on their elements. ${ }^{3 \mathrm{I}}$ So a committed structuralist should resist the idea that the dependence in question is at all essential to a foundationally-informed conception of set. ${ }^{32}$ And this is indeed the view taken by Parsons, who thinks that the iterative conception is just "part of an explanation by analogies, and not necessarily part of the literal truth about sets" (I995: 79).

But I think there's good evidence that the iterative conception is not only used as an analogy, or at least that it wasn't always used as such. Gödel, for instance, invokes it to motivate axioms which, he claims, "only unfold the content of the concept of set" (I964: 26I). The following passage nicely illustrates his view:

[T] he axioms of set theory by no means form a system closed in itself, but, quite on the contrary, the very concept of set [i.e. the iterative conception of the universe of sets] on which they are based suggests their extension

not as these terms are used in mathematical practice. For an illuminating discussion of these notions, see Tappenden (2005; 20I 2 ).

3I. A similar point is made in Linnebo (2008: 72). It's been argued by Incurvati (20I2) that this notion of dependence isn't a necessary component of the iterative conception. I won't dispute this-for my purposes it will be enough that some notion of dependence mattered to Gödel's take on the iterative conception. And though Incurvati denies it, I think there is good evidence it did. For instance, Gödel remarks that the iterated powerset operation "is to be understood so as to include also transfinite iteration, the totality of sets obtained by finite iteration forming again a set and a basis for a further application of the operation" (I964: 259nI 3, emphasis mine). Gödel may not have had any robust notion of dependence in mind here, but this is nonetheless more than a structuralist can allow.

32. Structuralists might agree that the identity of sets is determined by the membership relation they bear to each other, but on their view this membership relation is itself only given formally, by describing which objects it relates to which. The resulting kind of membership relation is very thin, and wouldn't account for the kind of dependence suggested by the iterative picture (even if one doesn't understand it in metaphysical terms). 
by new axioms which assert the existence of still further iterations of the operation "set of." (I964: 260)

So for Gödel, ZFC doesn't exhaust the conception of the universe of sets provided by the iterative account, and the conception itself suggests axioms which would complement ZFC in a natural manner. And it seems that he viewed the kind of justification provided by such an account as especially robust, referring as he does to the "intrinsic necessity" of the resulting axioms (I964: 26I). Whatever one makes of Gödel's intrinsic necessity, it seems clear that the iterative conception is not merely being used as an analogy or heuristic device-the conception actually describes the universe of sets, and provides a richer conception of it than what's codified in ZFC.

But on the structuralist view of sets, it's not clear what this richer conception could possibly tell us. For a structuralist, sets are essentially just positions in the structure of the set-theoretic universe-that is, as Parsons puts it, positions in the structure common to any "domain of 'objects' related in a relation called 'membership' satisfying conditions that can then be stated in the language of set theory," for instance the conditions stated by the ZFC axioms (I995: 75). ${ }^{33}$ So, on the reading of "essentially just" under consideration, all the foundationally-relevant information concerning sets should be exhausted by describing their role in this structure. What then are we to make of a conception of the universe of sets richer than the one afforded by ZFC? For a structuralist this conception shouldn't play any serious mathematical role, but this is clearly at odds with the use to which Gödel puts it.

This is another case, then, where mathematical practice doesn't seem to conform with the structuralist hypothesis. For Gödel invokes the iterative conception for justificatory purposes in a foundational context, and this conception goes beyond a purely structural characterization of sets. Here too, one might deny that Gödel truly isolated any essential features of sets, or one might deny that his remarks should be taken too seriously, or deny that he was really speaking from a "sophisticated" foundational standpoint. But without further argument about what qualifies as a sophisticated foundational standpoint, I take it this is just to give up on a deflationary interpretation of essentialist talk, which seeks to identify essential mathematical features as those which matter to the practice of mathematics. ${ }^{34}$

33. There's an additional difficulty here, since we have no second-order categorical characterization of ZFC at our disposal on par with the second-order Peano axioms for arithmetic, and so it may not be clear what the structure in question might be. Presumably the structuralist will invoke second-order ZFC, and invoke the quasi-categoricity of ZFC models, that is, the fact that for any two models of ZFC, either these models are isomorphic or one is isomorphic to an initial segment of the other. See Martin (200I) for an argument that quasi-categoricity of this sort is sufficient to rule out any damaging indeterminacy about the set theoretic universe.

34. In fact I think Gödel's case suggests a broader worry than this. For it's well known that $\mathrm{ZFC}$ is unable to settle a range of questions concerning sets (the most famous example being the 


\section{Saving Structuralism}

I've argued so far that there are serious difficulties involved in spelling out the structuralist position. It's crucial to this position that structural properties be singled out as the ones essential to mathematical objects, but there's no good way to articulate an adequate notion of essence, refusing to articulate it won't do, and more modest solutions aren't as well supported by mathematical practice as its proponents seem to assume.

But if the structuralist ontology is so problematic, how are we to respond to Benacerraf's problem? And what are we to make of the indifference mathematicians typically display towards questions concerning the nonstructural identity of the objects they study? In what follows I'm going argue that Benacerraf's problem was in fact rather poor motivation for a structuralist ontology. Then I'm going to sketch a form of structuralism which might do justice to the indifference in question without incurring the difficulties surveyed thus far. ${ }^{35}$

\section{I. Benacerraf Revisited}

Recall the structuralist's dissolution of Benacerraf's problem: it's true that there's nothing more to mathematical objects than the structural relations they bear to each other. But they should count as objects nonetheless, for positions in abstract structures are objects. So the fact that numbers can't be identified with any particular system of objects ordered in a successor-like fashion is no problem at all: they can be identified with a position in the abstract structure exemplified by all these systems of objects.

One might object to this response by pressing the Benacerrafian thought one step further. For given any structure which preserves the abstract form of a system of objects related to each other by a successor-like relation, other structures will exist which can do the job equally well. Hellman puts the point nicely:

continuum hypothesis), and it's a much-debated question which axioms one should add in order to supply this deficiency. It isn't clear what a structuralist would have to say about such debates. If there's nothing more to sets (or nothing more of foundational relevance) apart from the properties they possess in virtue of being the sorts of objects that satisfy the constraints of ZFC, what considerations could a principled argument for new set-theoretic axioms beyond ZFC possibly invoke? The structuralist position makes it hard to see how one might rationally decide on any new structure, given that the nature of sets is meant to be exhausted by describing the old. Burgess seems to share this concern (2009: 25).

35. This sketch will follow a suggestion mentioned in Burgess (2009: 20-2I). I won't engage here with other alternatives to the structuralist ontology, like the sort of eliminative structuralism I set aside at the beginning of this paper. This is because I've been convinced by the main line of criticism voiced against such alternatives, which is (roughly) that they require substantial metaphysical assumptions, that these assumptions get more substantial the more mathematics one attempts to recover, and that this casts some doubt on the gains achieved by avoiding reference to purely structural objects. A precise expression of this criticism can be found in Parsons (2008: ch. 3). 
[I]f we suppose, for the sake of argument, that an ante rem progression, $\langle N, \phi, \mathrm{I}\rangle$ [i.e. the structure of an $\omega$-sequence] is somehow attained, we immediately see that indefinitely many others, explicitly definable in terms of this one, qualify equally well as candidates to serve as the referents of our numerals. We need only permute, say, a finite number of elements of $N$, for example, the first two items, defining $\phi^{*}$ from $\phi$ in the obvious way. (200I: I95)

As Hellman notes, the resulting structure will work just as well as the first (after all, it will itself be the structure of an $\omega$-sequence). So, one might press on, which structure is it in which we find the number 2 ? There seems to be no particular structure whose position we can point to, which leads us back to the initial difficulty.

The structuralist response at this point is somewhat mad: the two structures in question are the same. ${ }^{36}$ In other words, there is no question of identifying a position in one particular structure rather than any of its isomorphic counterparts, for there really are no isomorphic counterparts to begin with-only one structure has the form of an $\omega$-sequence. Now, it's worth noting that the structures in question are certainly not identical if we regard them as mathematical objects-that is, permuting the first two items in a mathematical $\omega$-sequence would yield two distinct sequences, just as Hellman points out in his objection. So the claim that isomorphic structures are identical is not something which follows from our usual understanding of structures, but rather from a stipulation of identity conditions proper to the structuralist view.

But if we're prepared to make stipulations like these there's a much quicker solution to Benacerraf's problem. For if we aren't troubled by the thought that isomorphic structures are identical, it's hard to see what would be troubling about the fact that $2=\{\varnothing,\{\varnothing\}\}$ under some set-theoretic interpretation of the natural numbers, while $2=\{\{\varnothing\}\}$ under another. After all, one could simply stipulate that both of these interpretations are identical, and furthermore that any interpretation of the natural numbers as an $\omega$-sequence of sets is one and the same. On this rival stipulation two interpretations would be the same even when the specific $\omega$ sequences of sets assigned to numbers are not, much as, on the structuralist suggestion, two structures are the same even though their specific positions may not be.

36. Shapiro, for instance, claims we should simply "stipulate that two structures are identical if they are isomorphic" (I997: 93), and Resnik suggests a similar line, though he's generally reluctant to speak of identity relations between structures (I997: 209-I I). Parsons (to his credit, I think) does not suggest structuralism as a response to Benacerrafian worries of this sort. Shapiro seems to have backed off from his stipulation in later writings-for instance, in Shapiro (2006: I43) he claims that he "cannot rule out the hypothesis that there is more than one ante rem structure isomorphic to the natural numbers." If this is right, however, structuralism simply hasn't answered Benacerraf's challenge, since it hasn't told us which of these structures contains the positions our numerals are supposed to denote. 
Now, it's surely odd to claim that such interpretations are identical, given that they assign different sets to each natural number. But I submit that this is no more odd than stipulating that isomorphic structures are identical: in both cases, the demand for some specific system of objects to associate with the natural numbers is dismissed by deciding that, at some level, isomorphism is identity-in the structuralist case we stipulate that structures are identical when isomorphic, while on the view I'm presenting here we stipulate that interpretations are identical when they invoke isomorphic systems of sets. Benacerraf's problem stems from the existence of multiple interpretations of the natural numbers, with no principled way to choose between them. But if the structuralist is prepared to stipulate identity conditions to escape this problem at the level of structures, I don't see why she wouldn't introduce such conditions from the start, for the very interpretations which are supposed to motivate the structuralist ontology. 37

In fact there's a more sensible response to Benacerraf's worry than this. For the existence of multiple interpretations of various mathematical domains to set theory only becomes a problem if one takes these interpretations to reveal something important about the nature of the objects in that domain. But it's not clear why we should think this, nor did mathematicians ever take themselves to be producing a final mathematical ontology by identifying numbers or groups with various sets. So it's hard to see why one would be warranted in drawing any ontological conclusion on the basis of set theoretic interpretations of some mathematical domainwhether the conclusion be that there are no mathematical objects, or that the nature of these objects is structural.

In what follows I'll be spelling out what I think these equivalent interpretations $d o$ reveal, if we deny them any special ontological significance. My point for now is that even if one does feel the pull of Benacerraf's problem, the structuralist ontology only provides an answer to it by stipulating that, for structures, isomorphism is identity. And if one is prepared to accept mad stipulations of this sort, Benacerraf's problem can be dissolved without invoking any kind of mathematical ontology-simply stipulating the right identity conditions on interpretations would suffice. If this is right, the philosophical benefits of structuralism identified at the start of this paper are illusory.

\subsection{Structuralism Without Ontology}

Yet even once we set aside the Benacerrafian motivation for structuralism, it might seem that the view provides a good explanation for certain key features of mathe-

37. How is the solution any different? The structuralist escapes the problem because the question "in which structure do we really find the number 2 ?" turns out to be misguided (by fiat, there is only one structure with the right form). On the alternative stipulation, we escape the problem because the question "on which interpretation do we really find the number 2 ?" is misguided (by fiat, there is only one interpretation with the right form). 
matical practice. For mathematicians are, by and large, indifferent to nonstructural features of the objects they study, and the structuralist claim that mathematical objects are fundamentally structural entities seems to provide a good explanation for their indifference.

I think this insight is worth preserving, but I also think it can be preserved without making any problematic ontological claims. Here's what I want to suggest: questions concerning the nonstructural features of mathematical objects are typically dismissed, and often sound misguided, because their resolution rests on considerations too vague or too contentious to be accepted as part of ordinary mathematics. This doesn't reflect any deep fact about the nature of mathematical objects - at best it reflects facts about the scope and ambitions of ordinary mathematics, and perhaps about the manner in which various intellectual tasks are divided among mathematicians. Structural features are significant not because they constitute the nature of mathematical objects, but because they characterize objects in a manner acceptable to mathematicians working with potentially different conceptions of their nature.

Before spelling out the view a bit further, I want to clarify a few points. For you might wonder what counts as "ordinary" mathematics here, and how the "contentiousness" at stake differs from the contentiousness of mathematical questions ordinary mathematicians do care about resolving.

By "ordinary" mathematics, I mean the kind of research most mathematicians engage in every day-research that primarily involves proving theorems about mathematical objects of some kind or another from an accepted background theory (ZFC being the current standard). ${ }^{8}$ This is to be distinguished from the kind of foundational mathematics which focuses on the background theory itself, and in particular with its adequacy as a background theory. 39 One key feature of ordinary mathematics is the universality of its results: a mathematician who proves some theorem proves it for everyone, and establishes it once and for all. There may be some disagreement about which theorems are worthwhile or interesting, but the theorems themselves are not up for debate. Of course foundational arguments often invoke the theorems we can derive from a suggested set of axioms, and their status as theorems is no more up for grabs than it is in ordinary mathematics. In the foundational case, however, the axioms themselves might be disputed, as might be the criteria used to assess them-and there's no well-accepted arena to resolve these disputes which might play a role analogous to that of ZFC for ordinary mathematics. ${ }^{4}$

38. This is not to say that mathematicians work or think in set theoretic terms, only that "having a proof from ZFC" is an agreed upon criterion for being an acceptable mathematical result.

39. I think Weyl and Gödel provide a good example of this sort of foundational mathematics in the passages cited above (sections 3.3.I and 3.3.2).

40. Not anything goes, of course-the point is only that it's much harder to say what counts as resolving a foundational question than it is to say what counts as resolving a strictly mathematical 
In ordinary mathematics of this sort, then, though there might be propositions whose truth-values are vehemently disputed, it's always clear what it would take to resolve a dispute. Questions concerning the nonstructural nature of mathematical objects, by contrast, are contentious in a much less tractable way. It's hardly clear what it would take to resolve them, and the assumptions behind any strategy to do so are unlikely to meet with universal assent. So it's no surprise ordinary mathematicians would ignore these questions: the universality of their theorems would be lost if they were taken to be contingent on some position concerning the nonstructural nature of the objects they refer to. Still, none of this implies that there is no such nature to be investigated. ${ }^{4}$

Now, one might object that there being no such nature remains the most plausible explanation for the disregard of ordinary mathematicians. For it's not immediately clear what else would explain their disregard-unless of course we're meant to think that ordinary mathematicians are blithely setting aside contentious questions to preserve the universality of their results. If they can't be said to have isolated the nature of their objects, how might ordinary mathematicians be justified in setting aside questions concerning their nonstructural features?

To this I reply that there is no reason to think that the focus of ordinary mathematicians is arbitrary, and that refusing to take a stance on ontological matters does not commit us to the view that structural characterizations are mere dogmatism on their part. The account I've sketched so far is ontologically noncommittal: it allows for the possibility that mathematical objects be fundamentally structural, and for the possibility that they have a richer, nonstructural nature. I've raised some difficulties with the first of these possibilities in this paper, but having no concrete ontological counterproposal I won't advocate the second. This is all consistent with the view that the features of an $\omega$-sequence somehow reflect a deep truth about the nature of the natural numbers, and that in general the features of interest to ordinary mathematicians are among those which constitute part of the essence of the objects they study. It's only once we identify them with these objects' essences that we encounter the problems surveyed above. $4^{2}$

one. I'm only claiming here that questions concerning the nonstructural features of mathematical objects are too contentious for ordinary mathematics-I won't take a stand in this paper on whether they are too contentious for the purposes of foundational mathematics, or indeed what their relationship is to such mathematics. A similar take on the role of mathematical axioms is developed in more detail in Easwaran (2008).

4I. In particular, it doesn't imply that nonstructural features are never mathematically significant, or that "nothing mathematics countenances would fix [facts about nonstructural features]" (Resnik I997: 270). This would only be the case if we thought mathematics was nothing but ordinary mathematics, in the sense outlined above-and this is surely taking too narrow a view of the discipline.

42. The fact that mathematical objects don't have merely structural natures need not imply the stronger claim that mathematical objects have extrastructural natures: it might be a mistake to think 
In any case, I take it the main reason ordinary mathematicians feel confident in ignoring certain questions about mathematical objects is the same as in any other science, namely, that they trust the judgment of the mathematical community when it assents to purely structural characterizations and leaves further questions for specialists to work out. A parallel might be drawn with the status of fundamental concepts like mass, time, or space in modern physics. For the most part working physicists will be indifferent to questions concerning what mass really is-issues concerning the nature of mass rarely make their way into the work of nonspecialists, and physicists will typically rest content with a characterization of mass that focuses on its role in physical theories, for example as it appears in equivalences like $F=m a$, or $E=m c^{2}$. It seems to me they're perfectly justified in doing so, but that it would be a mistake to conclude that there's nothing more to say about mass once we've described the relations it bears to certain other physical concepts like force, energy, and so on, or that the nature of mass is only determined by the role it plays in the equations of physicists. 43

In fact, to end on a historical note, I think such an outlook is faithful to the spirit in which structural characterizations were initially conceived. Consider for instance what Dedekind has to say about his arithmetized characterization of the continuum (using what we now call "Dedekind cuts"):

The above comparison of the domain $R$ of rational numbers with a straight line has led to the recognition of the existence of gaps, of a certain incompleteness or discontinuity of the former, while we ascribe to the straight line completeness, absence of gaps, or continuity. In what then does this continuity consist? Everything must depend on the answer to this question, and only through it shall we obtain a scientific basis for the investigation of all continuous domains. By vague remarks upon the unbroken connection in the smallest parts obviously nothing is gained; the problem is to indicate a precise characteristic of continuity that can serve as the basis for valid deductions. For a long time I pondered over this in vain, but finally I found what I was seeking. This discovery will, perhaps, be differently estimated by different people; the majority may find its substance very commonplace. (I90I: IO-II, emphasis in the original)

So Dedekind took himself to be searching for a "scientific basis" for the study of

of mathematical objects as having "natures" to begin with. My argument here leaves open this more radical suggestion.

43. One might still hold such an "operational" view of mass for other reasons-my claim here is only that it's a mistake to hold it merely because we think ordinary physicists are right to adopt an operationalist outlook in their work. A good overview of recent debates about the nature of mass can be found in Jammer (2000). 
real numbers, whose primary purpose would be to provide "a precise characteristic of continuity that can serve as the basis for valid deductions." His primary purpose wasn't to characterize the nature of continuity, but rather to set aside "vague remarks" on the topic and focus on the features necessary to its mathematical investigation.

Note, moreover, that Dedekind takes his characterization of the continuum to be commonplace - an attitude well illustrated by the passage following his description of Dedekind cuts:

As already said I think I shall not err in assuming that every one will at once grant the truth of this statement; the majority of my readers will be very much disappointed in learning that by this commonplace remark the secret of continuity is to be revealed. (I90I: I I)

I take it this is good evidence that Dedekind was not seeking here to supplant existing conceptions of the nature of the continuum with his own. Rather, he was seeking an arithmetized characterization of the continuum because it would serve as an acceptable starting point regardless of one's conception of infinitesimals, and would therefore allow for a development of analysis unfettered by disputes concerning the nature of space.

My view is basically that Dedekind had it right. If we want to engage in ordinary, effective theorem-proving activities, which is what interests most mathematicians, it seems necessary to set aside questions having to do with the nonstructural aspects of mathematical objects. But this doesn't imply that these objects are essentially structural. All it reveals is that mathematicians working with various conceptions of the objects they study-or with no view at all about what mathematical objects are essentially — can agree on a structural characterization for their everyday work, and leave further issues (if any) for others to work out. This is sufficient to explain the key structuralist insights, or at least the key structuralist insights that are worth preserving.

\section{Conclusion}

I've argued in this paper against a common ontological version of structuralism, and I've attempted to present an ontologically neutral alternative. On this alternative view, the hermeneutic structuralist insights are not explained by the nature of mathematical objects, but rather by the manner in which mathematical research is organized: questions concerning the nonstructural features of mathematical objects are often dismissed because they are too contentious or vague to be considered part of ordinary mathematics. One philosophical benefit of the structuralist 
ontology, namely the solution it offered to Benacerraf's problem, was lost. But I've tried to cast some doubt on the merits of the solution structuralism was meant to provide.

The view I've defended leaves open a number of questions concerning the relationship between the nature of mathematical objects and the structural characterizations most mathematicians are happy to work with. What exactly is the status of nonstructural features? In what sense, for instance, did Weyl consider it a crucial part of the foundation of the theory of elliptical functions that they be embedded in Riemann surfaces? And what is it that makes structural characterizations so effective-why is there not more disagreement among working mathematicians about which of two structural equivalents is superior?

A position that leaves such questions unanswered might seem too feeble to merit the title of structuralism. I am happy to give up the title. My claim is only that the insights motivating common structuralist views can be satisfyingly explained without any contentious ontology.

\section{Acknowledgements}

Many thanks to Warren Goldfarb, Ned Hall, Peter Koellner, Michaela McSweeney, Bernhard Nickel, Zeynep Soysal, Amanda Wingate, members of Harvard's M\&E Workshop, and two anonymous referees for their helpful discussion and advice on various drafts of this paper.

\section{References}

Bell, John L. (I98I). Category Theory and the Foundations of Mathematics. British Journal for the Philosophy of Science, 32(4), 349-3 58. http://dx.doi.org/10.1093/bjps/32.4.349

Bell, John L. (1986). From Absolute to Local Mathematics. Synthese, 69(3), 409-426. http://dx.doi.org/10.1007/BF00413980

Benacerraf, Paul (I965). What Numbers Could Not Be. The Philosophical Review, 74(I), 47-73. http://dx.doi.org/10.2307/2183530

Burgess, John (1999). Book Review: Stewart Shapiro. Philosophy of Mathematics: Structure and Ontology. Notre Dame Journal of Formal Logic, 40(2), 283-291. http:// dx.doi.org/10.1305/ndjfl/1038949543

Burgess, John (2009). Putting Structuralism in Its Place. Unpublished manuscript.

Button, Tim (2006). Realistic Structuralism's Identity Crisis: A Hybrid Solution. Analysis, 66(3), 2 I 6-222. http://dx.doi.org/10.1093/analys/66.3.216

Dedekind, Richard ( I 888). Was Sind und Was Sollen die Zablen? Vieweg.

Dedekind, Richard (I90I). Essays on the Theory of Numbers (Wooster Woodruff Beman, Trans.). The Open Court Publishing Company.

Dummett, Michael (I99I). Frege: Philosophy of Mathematics. Harvard University Press. 
Easwaran, Kenny (2008). The Role of Axioms in Mathematics. Erkenntnis, 68(3), 38I39I. http://dx.doi.org/10.1007/s10670-008-9106-1

Fine, Kit (I994). Essence and Modality. Philosophical Perspectives, 8, I-I6.

Fine, Kit (200I). The Question of Realism. Philosopher's Imprint, I(I), I-30.

Gödel, Kurt (I 964). What Is Cantor's Continuum Problem? In Solomon Feferman, John W. Dawson Jr, Stephen C. Kleene, Gregory H. Moore, Robert M. Solovay, and Jean van Heijenoort (Eds.), Kurt Gödel, Collected Works Volume II: Publications 1938-1974, 254-270, Oxford University Press.

Hale, Bob (1996). Structuralism's Unpaid Epistemological Debts. Philosophia Mathematica, 4(2), I 24-47. http://dx.doi.org/10.1093/philmat/4.2.124

Hellman, Geoffrey (200I). Three Varieties of Mathematical Structuralism. Philosophia Mathematica, 9(2), I 84-2I I. http://dx.doi.org/10.1093/philmat/9.2.184

Hellman, Geoffrey (2005). Structuralism. In Stewart Shapiro (Ed.), The Oxford Handbook of Philosophy of Mathematics and Logic, 536-562, Oxford University Press.

Incurvati, Luca (20I2). How to Be a Minimalist About Sets. Philosophical Studies, I 59(I), 69-87. http://dx.doi.org/10.1007/s11098-010-9690-1

Jammer, Max (2000). Concepts of Mass in Contemporary Physics and Philosophy. Princeton University Press.

Keränen, Jukka (200I). The Identity Problem for Realist Structuralism. Philosophia Mathematica, 9(3), 308-330. http://dx.doi.org/10.1093/philmat/9.3.308

Keränen, Jukka (2006). The Identity Problem for Realist Structuralism II: A Reply to Shapiro. In Fraser MacBride (Ed.), Identity and Modality, I46-I63, Oxford University Press.

Ketland, Jeffrey (2006). Structuralism and the Identity of Indiscernibles. Analysis, 66(4), 303-3 I 5. http://dx.doi.org/10.1093/analys/66.4.303

Ladyman, James (2005). Mathematical Structuralism and the Identity of Indiscernibles. Analysis, 65(3), 21 8-22I. http://dx.doi.org/10.1093/analys/65.3.218

Leitgeb, Hannes, and James Ladyman (2008). Criteria of Identity and Structuralist Ontology. Philosophia Mathematica, I6(3), 288-396.

Linnebo, Øystein (2008). Structuralism and the Notion of Dependence. Philosophical Quarterly, 58(230), 59-79.

Martin, Donald A. (200I). Multiple Universes of Sets and Indeterminate Truth Values. Topoi, 2o(I), 5-I6. http://dx.doi.org/10.1023/A:1010600724850

Parsons, Charles (I990). The Structuralist View of Mathematical Objects. Synthese, 84(3), 303-346. http://dx.doi.org/10.1007/BF00485186

Parsons, Charles (I 995). Structuralism and the Concept of Set. In Walter Sinnott-Armstrong, Diana Raffman, and Nicholas Asher (Eds.), Modality, Morality, and Belief: Essays in Honor of Ruth Barcan Marcus, 74-92, Cambridge University Press.

Parsons, Charles (2004). Structuralism and Metaphysics. Philosophical Quarterly, 54(2 I 4), 56-77. http://dx.doi.org/10.1111/j.0031-8094.2004.00342.x

Parsons, Charles (2008). Mathematical Thought and Its Objects. Cambridge University Press.

Resnik, Michael (I98I). Mathematics as a Science of Patterns: Ontology and Reference. Nô̂s, I 5(4), 529-5 50. http://dx.doi.org/10.2307/2214851

Resnik, Michael (I997). Mathematics as a Science of Patterns. Oxford University Press.

Rosen, Gideon (2010). Metaphysical Dependence: Grounding and Reduction. In Bob Hale and Aviv Hoffman (Eds.), Modality: Metaphysics, Logic, and Epistemology, 109-1 36 , Oxford University Press. 
Shapiro, Stewart (1997). Philosophy of Mathematics: Structure and Ontology. Oxford University Press.

Shapiro, Stewart (2006). Structure and Identity. In Fraser MacBride (Ed.), Identity and Modality, I09-I45, Oxford University Press.

Shapiro, Stewart (2008). Identity, Indiscernibility, and Ante Rem Structuralism: The Tale of $i$ and -i. Philosophia Mathematica, I6(3), 28 5-309. http://dx.doi.org/10.1093/philmat/nkm042

Tappenden, Jamie (2005). Proof Style and Understanding in Mathematics I: Visualization, Unification and Axiom Choice. In Paolo Mancosu, Klaus F. Jørgensen, and Stig A. Pedersen (Eds.), Visualization, Explanation and Reasoning Styles in Mathematics, I47$2 \mathrm{I}_{4}$, Springer.

Tappenden, Jamie (20I2). Fruitfulness as a Theme in the Philosophy of Mathematics. Journal of Philosophy, I09(I-2), 204-2I9.

Weaver, George (I998). Structuralism and Representation. Philosophia Mathematica, 6(3), 257-27I. http://dx.doi.org/10.1093/philmat/6.3.257

Weyl, Hermann (I9I3). Die Idee der Riemannschen Fläche. Teubner.

Wilson, Mark (I992). Frege: The Royal Road from Geometry. Nô̂s, 26(2), I49-1 80. 